\title{
Inflationary freedom and cosmological neutrino constraints
}

\author{
Roland de Putter, ${ }^{1,2}$ Eric V. Linder, ${ }^{3,4}$ and Abhilash Mishra ${ }^{2}$ \\ ${ }^{1}$ Jet Propulsion Laboratory, California Institute of Technology, Pasadena, California 91109, USA \\ ${ }^{2}$ California Institute of Technology, Pasadena, California 91125, USA \\ ${ }^{3}$ Berkeley Lab \& Berkeley Center for Cosmological Physics, University of California, \\ Berkeley, California 94720, USA \\ ${ }^{4}$ Institute for the Early Universe WCU, Ewha Womans University, Seoul 120-750, Korea
}

(Received 5 February 2014; published 1 May 2014)

\begin{abstract}
The most stringent bounds on the absolute neutrino mass scale come from cosmological data. These bounds are made possible because massive relic neutrinos affect the expansion history of the universe and lead to a suppression of matter clustering on scales smaller than the associated free streaming length. However, the resulting effect on cosmological perturbations is relative to the primordial power spectrum of density perturbations from inflation, so freedom in the primordial power spectrum affects neutrino mass constraints. Using measurements of the cosmic microwave background (CMB), the galaxy power spectrum and the Hubble constant, we constrain neutrino mass and number of species for a modelindependent primordial power spectrum. Describing the primordial power spectrum by a 20-node spline, we find that the neutrino mass upper limit is a factor 3 weaker than when a power law form is imposed, if only CMB data are used. The primordial power spectrum itself is constrained to better than $10 \%$ in the wave vector range $k \approx 0.01-0.25 \mathrm{Mpc}^{-1}$. Galaxy clustering data and a determination of the Hubble constant play a key role in reining in the effects of inflationary freedom on neutrino constraints. The inclusion of both eliminates the inflationary freedom degradation of the neutrino mass bound, giving for the sum of neutrino masses $\Sigma m_{\nu}<0.18 \mathrm{eV}$ (at $95 \%$ confidence level, Planck + BOSS $+H_{0}$ ), approximately independent of the assumed primordial power spectrum model. When allowing for a free effective number of species, $N_{\text {eff }}$, the joint constraints on $\Sigma m_{\nu}$ and $N_{\text {eff }}$ are loosened by a factor 1.7 when the power law form of the primordial power spectrum is abandoned in favor of the spline parametrization.
\end{abstract}

DOI: $10.1103 /$ PhysRevD.89.103502

PACS numbers: 98.80.Es, 14.60.Pq, 98.80.Cq

\section{INTRODUCTION}

Cosmology has revealed rich structure beyond the Standard Model of particle physics, with dark matter, an inflationary acceleration of expansion in the early universe and a dark energy acceleration in the late universe. Moreover, we now know that a required extension to the standard models of both particle physics and cosmology is the presence of neutrino mass. Laboratory neutrino oscillation experiments indicate that at least two species must be massive, and the sum of all three species must be $\Sigma m_{\nu}>$ $0.055 \mathrm{eV}[1]$.

The strongest upper bounds arise from cosmological measurements sensitive to the suppression of matter density perturbations caused by neutrino free streaming and to the effect of neutrino mass on the expansion history of the universe. These bounds, however, start from assumptions about early universe physics, such as a power law form for the primordial power spectrum (PPS). Restricting the form in this way will tighten the neutrino limits and, if there exists deviation from a power law, bias the results. While a power law PPS is predicted by the simplest models of inflation, there exist other models of inflation with nontrivial features in the
PPS. As such, it is important to investigate the neutrino bounds without making assumptions about (unknown) inflationary physics.

The key cosmological observable (in linear perturbation theory) is the late-time power spectrum $P(k)$ (or angular power spectrum $C_{\ell}$ ), which is a convolution of the PPS $\Delta_{\mathcal{R}}^{2}(k)$ that encodes information about inflationary physics and the transfer function of cosmological perturbations. The transfer function can be calculated from well-tested physics (linearized gravity and photonbaryon fluid physics) and is described by a small number of cosmological parameters, including neutrino mass and effective number of species, specifying the energy budget and ionization history of the universe. The PPS on the other hand depends on physics at an energy scale that has never been directly tested. Usually, it is parametrized as a scale invariant power law inspired by the simplest models of inflation. However, assuming a particular functional form for the PPS can bias the estimates of cosmological parameters (e.g. [2,3]).

In this paper we focus on how the constraints on neutrino mass and effective number of species are affected when more freedom is allowed in the form of the PPS. Indeed, even enlarging the parameter space from 
a simple power law PPS to one with running (scale dependence) of the slope can strongly affect the neutrino bounds. For example, $[4,5]$ found significant covariance between running and both neutrino mass and effective number of species.

Due to the scale-dependent effect of neutrino mass on the perturbation power spectrum, properties of neutrinos and of the inflaton responsible for generating the PPS enter in the data tied closely together in ways different from other extensions to the Standard Model like dark matter and dark energy. Many inflation theories in the post-Planck data era do have spectral features deviating from a simple power law. These may include oscillations, steps, and bumps and can arise from physics such as axion monodromy [6], holography [7], sound speed [8], among many other current ideas.

Several approaches exist in the literature for reconstructing a free PPS. These include the "cosmic inversion" method [9-13], regularization methods like truncated singular value decomposition [14] and Richardson-Lucy iteration $[3,15,16]$, and maximum entropy deconvolution [17]. Recently the authors of [18] carried out a reconstruction of the PPS employing Tikhonov regularization using multiple data sets and detected several features in the PPS at a $2 \sigma$ level of significance (also compare [3]).

Here we focus on the issue of the dependence of neutrino constraints on assumptions about the PPS, rather than reconstructing the PPS per se. Suppression of growth due to massive neutrinos enters around the free streaming scale, or comoving wave numbers $k \gtrsim 0.01 \mathrm{Mpc}^{-1}$. Moreover, power spectra of cosmic perturbations are fundamentally observed as a function of angular scales (and redshifts, in case of three-dimensional large-scale structure), so that the effect of massive neutrinos on the expansion history can in principle cause changes in the observed CMB and large-scale structure power spectra on all scales by shifting these spectra horizontally. Variations in primordial power on all observable scales, from approximately the cosmic horizon scale, down to small scales where Silk damping (CMB) or nonlinear clustering (large-scale structure) degrades the cosmological information, may therefore affect neutrino constraints. Thus we investigate how freedom in the PPS over some 2.5 orders of magnitude, $k \approx 0.001-0.35 \mathrm{Mpc}^{-1}$, affects determination of the neutrino and cosmological parameters.

For our purposes of investigating effects on the cosmological parameters we want a robust, model-independent description. Examples include the use of wavelets [19-22], principal components [23], tophat bins with no interpolation [24], linear interpolation [25-31], smoothing splines [32-35], and power-law bins [36]. We choose to describe the PPS by cubic splines, following [37]. This preserves model independence while encompassing the power law model, and is smooth.
A completely free form PPS could exactly mimic (at least within cosmic variance) neutrino mass effects for a single type of observations, e.g. cosmic microwave background (CMB) temperature perturbations, though possibly requiring order unity sculpting of the PPS [2]. Since other observations, such as CMB polarization spectra or matter density power spectra, enter with different redshift-weighted transfer functions relative to the PPS, and since these transfer functions depend in different ways on cosmological parameters, then combining power spectra data (or external constraints on other cosmological parameters) plays an important role in fitting both inflaton and neutrino properties (see [38]).

We therefore carry out several studies on how freedom in the PPS affects neutrino constraints: we consider CMB data alone, with inclusion of large-scale structure data, and with inclusion of Hubble constant measurement. As well we investigate the standard scenario with three neutrinos with unknown total mass, and also the scenario with a free total mass and a free effective number of neutrino species, $N_{\text {eff }}$.

Section II discusses the treatment of the PPS in a substantially model-independent manner. We describe the data used and our method for calculating parameter constraints in Sec. III. Cosmological parameter estimation results are presented in Sec. IV, examining the covariance between standard, neutrino, and extended PPS parameters, and the role of $\mathrm{CMB}$, large-scale structure, and Hubble constant data. We summarize our results in Sec. V and discuss the sensitivity of our constraints to the assumed PPS spline parametrization and to the CMB data included in the Appendixes.

\section{PRIMORDIAL POWER SPECTRUM}

In the inflationary scenario for generation of density perturbations, the universe is in a near-de Sitter state where the quantum fluctuations of the inflaton field produce scalar (gravitational potential) and tensor (gravitational wave) metric perturbations. In simple, single field models of inflation the gravitational potential perturbations are Gaussian and nearly scale invariant. This implies that they (and the density perturbations through the Poisson equation) are completely characterized by the two-point function or power spectrum. Since inflation must end, the spacetime is not exactly de Sitter and so the power spectrum is not exactly scale invariant.

For slow rolling of the inflaton field value over time, the PPS is conventionally expanded in a Taylor series about the value at some pivot scale,

$$
\Delta_{\mathcal{R}}^{2}(k)=\Delta_{\mathcal{R}}^{2}\left(k_{0}\right)\left(\frac{k}{k_{0}}\right)^{n_{s}-1+\left(\alpha_{s} / 2\right) \ln \left(k / k_{0}\right)},
$$


where $\Delta_{\mathcal{R}}^{2}$ is the curvature perturbation power spectrum, $k_{0}$ the pivot wave number, $n_{s}$ the tilt, and $\alpha_{s}$ the running. However, in more general inflation scenarios the slow, smooth evolution of the field can be replaced with faster variations, oscillations, and features (which can in particular circumstances be treated through a generalized slow roll formalism [39]).

Forcing a power law form could bias the results for all quantities, even the late-time cosmological parameters, and certainly affects the uncertainty of their estimation. Given that current cosmological data are severely constraining the sum of neutrino masses from above, with this bound beginning to approach the lower limit imposed by neutrino oscillation terrestrial experiments, it is worthwhile exploring the link between inflationary assumptions and neutrino constraints.

Therefore we attempt a model-independent approach where no functional form is assumed. The values of the PPS amplitude at various wave numbers (nodes) are allowed to float freely, and these are smoothly connected using a cubic spline. With enough nodes this can give an excellent approximation to a wide range of functions, including nonmonotonic and oscillatory behavior. The typical number of nodes used in the literature is around 20 though it can be as high as 50 [40]; we use 20, although we explore the effects of using 10 or 40 in Appendix A. The number of nodes (parameters) is thus much less than the number of data points and a Markov chain Monte Carlo (MCMC) analysis is expected to give accurate confidence limits on cosmological parameters.

We first define a normalized primordial power spectrum $p(k)$, such that

$$
\Delta_{\mathcal{R}}^{2}(k) \equiv \Delta_{\mathcal{R}, 0}^{2} p(k)=\Delta_{\mathcal{R}, 0}^{2} \times \operatorname{spline}\left[p\left\{k_{i}\right\}\right]
$$

where we choose $\Delta_{\mathcal{R}, 0}^{2} \equiv 2.36 \times 10^{-9}$ (the approximate value of the primordial power spectrum amplitude preferred by current data), such that $p(k)$ is expected to be of order unity. Note that the actual amplitude can vary without loss of generality by changing $p(k)$. We then specify the values $p_{i} \equiv p\left(k_{i}\right)$ of this normalized PPS at a set of $N$ spline nodes, $k_{i}$. At $k<k_{1}$, we fix $p(k)=1$, whereas at $k>k_{N}$, we set $p(k)=p_{N}$. In the intermediate range, $p(k)$ is given by a cubic spline.

To encompass the range of scales well probed by CMB and galaxy clustering data we take $k_{1}=0.001 \mathrm{Mpc}^{-1}$ and $k_{N}=0.35 \mathrm{Mpc}^{-1}$. The low end is slightly larger than the wave vector corresponding to the cosmic horizon, and is thus close to the smallest $k$ that could be probed by any observable. For the spacing of the $k_{i}$ nodes we follow [37], using $N=20$ nodes with a logarithmic spacing, such that $k_{i+1}=1.36 k_{i}$. This allows the PPS to cause variations in the CMB and galaxy power spectra on scales comparable to those associated with the features (such as baryon acoustic oscillations) introduced by the transfer functions depending on cosmological parameters, and hence we can explore how PPS freedom interacts with cosmological parameter estimation. In Appendix A we will consider alternative choices of the PPS characteristics to test the robustness of the results.

We allow the PPS parameters $\left\{p_{i}\right\}$ to vary in the range $0.01<p_{i}<10$ (imposing uniform priors). When the node parameters are close to zero, it is possible for the spline to return negative values for $p(k)$ at some intermediate $k$. To avoid this unphysical behavior of the PPS, we restrict to $p(k) \geq 0.01$, setting $p(k)=0.01$ whenever the spline returns a value smaller than 0.01 . Our results are insensitive to the exact choice for the lower bound of the $p_{i}$ range and to the details of the cutoff because primordial power spectra with nodes $p_{i} \sim 0.01$ have a very low likelihood. Given the PPS, the CMB temperature and polarization power spectra and the matter power spectrum at any redshift are obtained by convolving the PPS with the transfer functions for CMB multipoles and matter density perturbations, in the usual manner.

\section{DATA AND METHOD}

Our choice of CMB data closely follows that of the Planck Collaboration [41,42]. We use the Planck temperature power spectrum, together with high resolution (high- $\ell$ ) temperature data from the Atacama Cosmology Telescope (ACT) $[43,44]$ and the South Pole Telescope (SPT) $[45,46]$. We also include low multipole $(\ell<23)$ polarization data from WMAP (referred to as WP in the Planck papers) [47]. In the standard case of a power law PPS, the latter data set mainly serves to constrain the optical depth due to reionization, $\tau$, which is otherwise strongly degenerate with the amplitude of primordial perturbations, $A_{s}$. For a free PPS, the polarization data play a more important role, as discussed in Appendix B. The temperature power spectrum measurements from Planck, ACT, and SPT are illustrated in Fig. 1 (see Sec. IV for more discussion of this figure).

We incorporate the required set of 31 "nuisance" parameters needed to take into account foregrounds, beams, and calibration uncertainties. These parameters will be marginalized over when parameter constraints are computed. The main use of the high- $\ell$ data is to help constrain a number of these nuisance parameters describing extragalactic foregrounds, thus improving constraints on cosmological parameters that are partially degenerate with the "nuisance" parameters when using Planck data only.

In order to keep the number of observables small and the interpretation of our results clean, we do not include the reconstructed lensing potential power spectrum data from Planck. However, the effect of gravitational lensing on the CMB temperature power spectrum is modeled and in fact contributes strongly to the constraints on neutrino mass in the case of a power law primordial power spectrum [41]. 


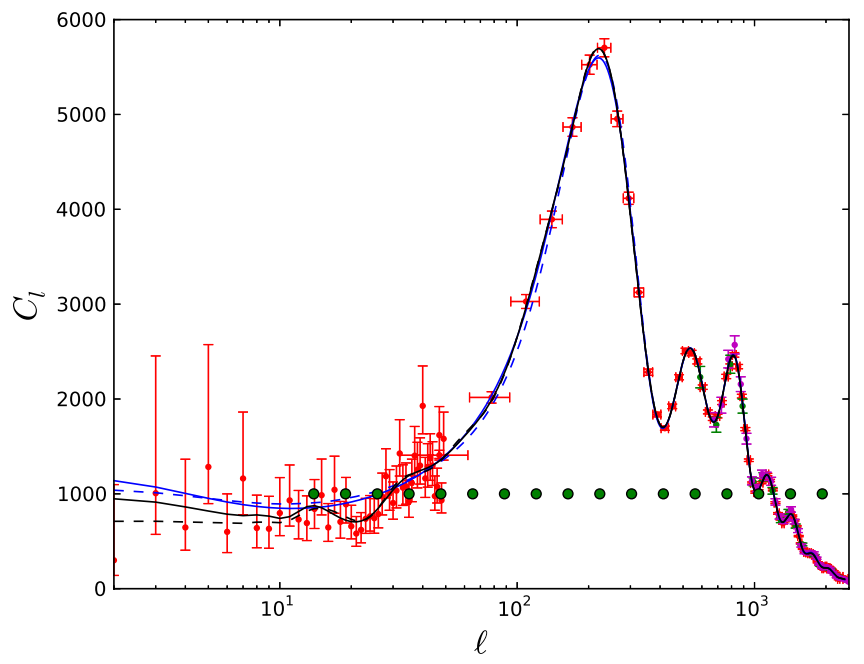

FIG. 1 (color online). The CMB temperature power spectrum is plotted with data points from Planck in red, from ACT in green and from SPT in magenta. The large green dots indicate the locations of the spline nodes. The blue (black) solid curve is the best-fit theory spectrum to the $C M B$ data set in the case of a power law (free/splined) PPS, for fixed $\Sigma m_{\nu}=0 \mathrm{eV}$. The respective dashed curves show the best fit spectra for fixed $\Sigma m_{\nu}=2.5 \mathrm{eV}$. The freedom in the spline PPS can compensate for the neutrino mass to keep the power spectrum at $\ell>10$ virtually the same as the zero mass case, showing how inflaton freedom affects neutrino constraints.

Physically, neutrino mass acts to suppress power in the photon or matter density perturbations, damping small scale power. Moreover, massive neutrinos affect the expansion history and thus cosmic distances, modifying the projection from physical scales to observed angles and redshifts. For a free form PPS one can imagine a complete degeneracy could arise between a power law primordial spectrum with some neutrino mass and an appropriate PPS with a different neutrino mass. A similar confusion could occur for the number of effective neutrino species, although this can also affect larger scales through changing the time of matter-radiation equality. However, since these are one time adjustments, measurements of the perturbation power spectrum with different redshift weightings would become distinguishable. Such different weights occur in the polarization, lensing, or matter power spectra relative to the temperature power spectrum.

One of the goals of this article is to see to what extent combining the high redshift measurement from the CMB with a low redshift measurement of large-scale structure can constrain neutrino properties (and other cosmological parameters) without making strong assumptions about the primordial power spectrum. The current state of the art in large-scale structure surveys is the Baryon Oscillation Spectroscopic Survey (BOSS) [48]. Therefore, we use the angle-averaged galaxy density power spectrum obtained from the BOSS CMASS sample (see, e.g.
[49,50]) made available with data release 9 (DR9, [51]). This sample has an effective redshift $z_{\text {eff }}=0.57$ and covers an effective volume $V_{\text {eff }}=2.2 \mathrm{Gpc}^{3}$. The observed power spectrum is shown in Fig. 5 (see Sec. IV for further discussion of this figure). We include only the black data points in our likelihood, corresponding to the wave vector range $k=0.03-0.12 h / \mathrm{Mpc}$.

In the likelihood analysis, we marginalize over possible systematic contributions to the large-scale power spectrum, by subtracting from the observed spectrum a template, $P_{g, \text { obs }}(k) \rightarrow P_{g, \text { obs }}(k)-S P_{\text {sys }}(k)$, where $P_{\text {sys }}(k)$ is the template and $S$ a free parameter with prior range $S=[-1,1]$. We refer to [52,53] for details. For each set of cosmological and nuisance parameters, we then compare the observed spectrum to a theory spectrum. Since we restrict the analysis to linear and only mildly nonlinear scales, it is appropriate (see e.g. [4,54]) to model this theory power spectrum using the simple "P-model,"

$$
P_{g}(k)=b^{2} P_{m}(k)+P_{0} .
$$

Here, $b$ is a free, scale-independent galaxy bias and $P_{m}(k)$ the linear matter power spectrum. The additional free parameter $P_{0}$ is included to describe deviations from scale-independent bias, which can arise from a combination of nonlinear galaxy bias, nonlinear redshift space distortions and stochastic bias. Finally, this theory spectrum is convolved with the survey window function to match the expectation value of the spectrum estimated from the data; see [4] for details. Our analysis of the BOSS power spectrum is identical to that in [55].

Finally, we consider the inclusion of a prior on the Hubble constant. We use the value $H_{0}=73.8 \pm$ $2.4 \mathrm{~km} / \mathrm{s} / \mathrm{Mpc}$ by [56] (R11) based on supernova distances, which are calibrated using Cepheids observed with the Hubble Space Telescope. We will refer to this measurement as HST. Since the CMB is mostly sensitive to the physical densities, while the matter clustering is more affected by the fractional densities, the Hubble constant can play an important role in linking the two. Moreover, the CMB data alone leave a strong anticorrelation between neutrino mass and $H_{0}$ so that the inclusion of a tight $H_{0}$ prior can be expected to have a significant effect on the neutrino mass bound.

While the $H_{0}$ measurement by R11 has been widely used in cosmological analyses, some caution is in order with regards to constraints based on this measurement. First of all, there is a significant and well-known tension between the above direct measurement and the value inferred from the Planck data in the context of the standard $\Lambda$ CDM model [41] (regardless of whether neutrino mass is a free parameter). While this might be a sign of new physics, such as the existence of additional relativistic species, it could also point to a problem with the data (analysis). Secondly, the R11 
Cepheid data have recently been reanalyzed [57] (taking into account the revised geometric maser distance to NGC4258 and modifying the treatment of outliers and Cepheid metallicity), leading to a non-negligible shift in the Hubble constant, $H_{0}=72.5 \pm 2.5 \mathrm{~km} / \mathrm{s} / \mathrm{Mpc}$. In Sec. IV C, we will therefore also present neutrino mass constraints using the revised $H_{0}$ measurement.

We modify CAMB [58] to use the set $\left\{p_{i}\right\}$ as a replacement for the usual amplitude $A_{s}$ and tilt $n_{s}$ (and running $\alpha_{s}$ ). We obtain both the CMB power spectra and the low redshift matter power spectrum (which in turn is used to compute the galaxy power spectrum) from this modified version of CAMB. In addition to the remaining four standard vanilla cosmology parameters of the physical baryon density $\omega_{b} \equiv \Omega_{b} h^{2}$, cold dark matter density $\omega_{c} \equiv \Omega_{c} h^{2}$, cosmological constant density $\Omega_{\Lambda}$ (or, equivalently, Hubble parameter $H_{0}$ ), and reionization optical depth $\tau$, we include parameters to describe the neutrino sector. We consider two scenarios: one where the effective number of neutrino species is fixed to the standard model value $N_{\text {eff }}=3.046$ and only the sum of neutrino masses $\Sigma m_{\nu}$ is a free parameter, and one where $N_{\text {eff }}$ is left free as well.

For the standard scenario with three species, we assume a degenerate hierarchy, with each neutrino having a mass $\Sigma m_{\nu} / 3$. With $N_{\text {eff }}$ free and $N_{\text {eff }}>3.046$, we simply add an effective number of $N_{\text {eff }}-3.046$ massless neutrino species to the three massive ones. When $N_{\text {eff }}<3.046$, we lower the temperature of the three standard neutrinos to obtain the energy density corresponding to $N_{\text {eff }}$. In this regime, the parameter $\Sigma m_{\nu}$ should be interpreted as a rescaled sum of neutrino masses, $\left(N_{\text {eff }} / 3.046\right)^{3 / 4} \Sigma m_{\nu}$.

Thus there are either 25 or 26 cosmological parameters overall, plus 34 ( 31 for the CMB and 3 for the galaxy power spectrum) "nuisance" parameters from the data. We will be particularly interested in the covariance between the PPS parameters and the neutrino parameters - that is, how much the relaxation of an assumed power law form influences the neutrino constraints. Modifying CosmoMC [59], we carry out a Markov chain Monte Carlo analysis for parameter estimation, sampling the cosmological parameters including PPS parameters, and the nuisance parameters.

Before moving on to the results, we note that, generally, the constraints on neutrino properties come both from the suppressed growth of cosmic perturbations and from the expansion history. The latter relates to cosmic distances $d(z)=\int d z / H(z)$ and the Hubble rate $H(z)$, which, through the Friedmann equation, is proportional to the (square root of the) total energy density of the universe. Since the present neutrino energy density is proportional to the sum of neutrino masses, $\omega_{\nu} \equiv \Omega_{\nu} h^{2} \approx \Sigma m_{\nu} / 94 \mathrm{eV}$, and since the relativistic neutrino energy density is proportional to $N_{\text {eff }}$, both cosmic distances and the Hubble rate probe neutrino mass.

\section{COSMOLOGICAL CONSTRAINTS}

\section{A. CMB-only constraints on neutrino mass}

We first consider constraints from the CMB-only combination of data sets. The temperature power spectrum measurements are shown in Fig. 1. For comparison, the solid black (spline PPS) and blue (power law PPS) curves show the best-fit theory power spectra for fixed $\Sigma m_{\nu}=0$. These are in excellent agreement on multipoles $\ell \gtrsim 60$, and the spline PPS has the freedom to fit variations in the data at smaller multipoles. The dashed curves then show the effect of $\Sigma m_{\nu}=2.5 \mathrm{eV}$. This noticeably changes the CMB power spectrum and hence can be ruled out in the power law PPS case at high ${ }^{1}$ significance. However, there can be enough freedom in general in the PPS to allow even such a large sum of neutrino masses, as shown by the agreement of the dashed and solid black (spline PPS) curves. This is reflected by the fact that, as we will see, $\Sigma m_{\nu}=2.5 \mathrm{eV}$ lies within the $99.9 \%$ C.L. region for the spline PPS (although outside the $95 \%$ C.L. region).

The green dots indicate the multipoles corresponding to the projected spline node wave vectors, $\ell_{i}=k_{i} D_{\mathrm{LSS}}$, where $D_{\text {LSS }}$ is the comoving distance to the CMB last scattering surface. While the mapping between $k$ and $\ell$ is in reality not one-to-one, i.e. power at a given wave vector $k$ translates to power at a range of multipoles $\ell$ rather than just $\ell_{i}$, the $\ell_{i}$ 's give an impression of where a given PPS node modifies the angular temperature power spectrum. Specifically, one sees that the PPS spline is flexible enough to affect the temperature spectrum across the full range of multipoles constrained by the data (the signal-to-noise in the multipoles below our lowest node, $\ell \lesssim 10$, is small), and to alter the spectrum on the scale of the acoustic oscillations, like late-time cosmological parameters.

Figure 2 shows that, despite the large freedom allowed in its shape, the CMB data place strong constraints on the PPS. The black points and error bars show the mean PPS values at the spline nodes and the uncertainties. The black curve represents the corresponding best-fit PPS at all $k$ (i.e. the cubic spline going through the nodes shown in the figure). These PPS constraints are obtained while simultaneously fitting for the cosmological parameters and the nuisance parameters associated with the CMB data. The dashed straight line in Fig. 2 depicts the power law PPS best fitting the CMB data set for comparison.

Except for the highest $k$ node $\left(k=0.35 \mathrm{Mpc}^{-1}\right.$, not shown in Fig. 2), all node powers are reasonably well constrained. The nodes in the range $k=0.009-0.26 \mathrm{Mpc}^{-1}$ all have $<$ $10 \%$ uncertainties (relative to the fiducial amplitude $\Delta_{\mathcal{R}, 0}^{2}=2.36 \cdot 10^{-9}$ ), with the best-constrained node (at $k=0.14 \mathrm{Mpc}^{-1}$ ) being measured with $3 \%$ precision. The

\footnotetext{
${ }^{1} \mathrm{~A}$ mass $\Sigma m_{\nu}=2.5 \mathrm{eV}$ is ruled out at (much) more than $3 \sigma$, but the exact significance is difficult to quantify as the chains do not contain any points beyond $\Sigma m_{\nu}=2.5 \mathrm{eV}$.
} 


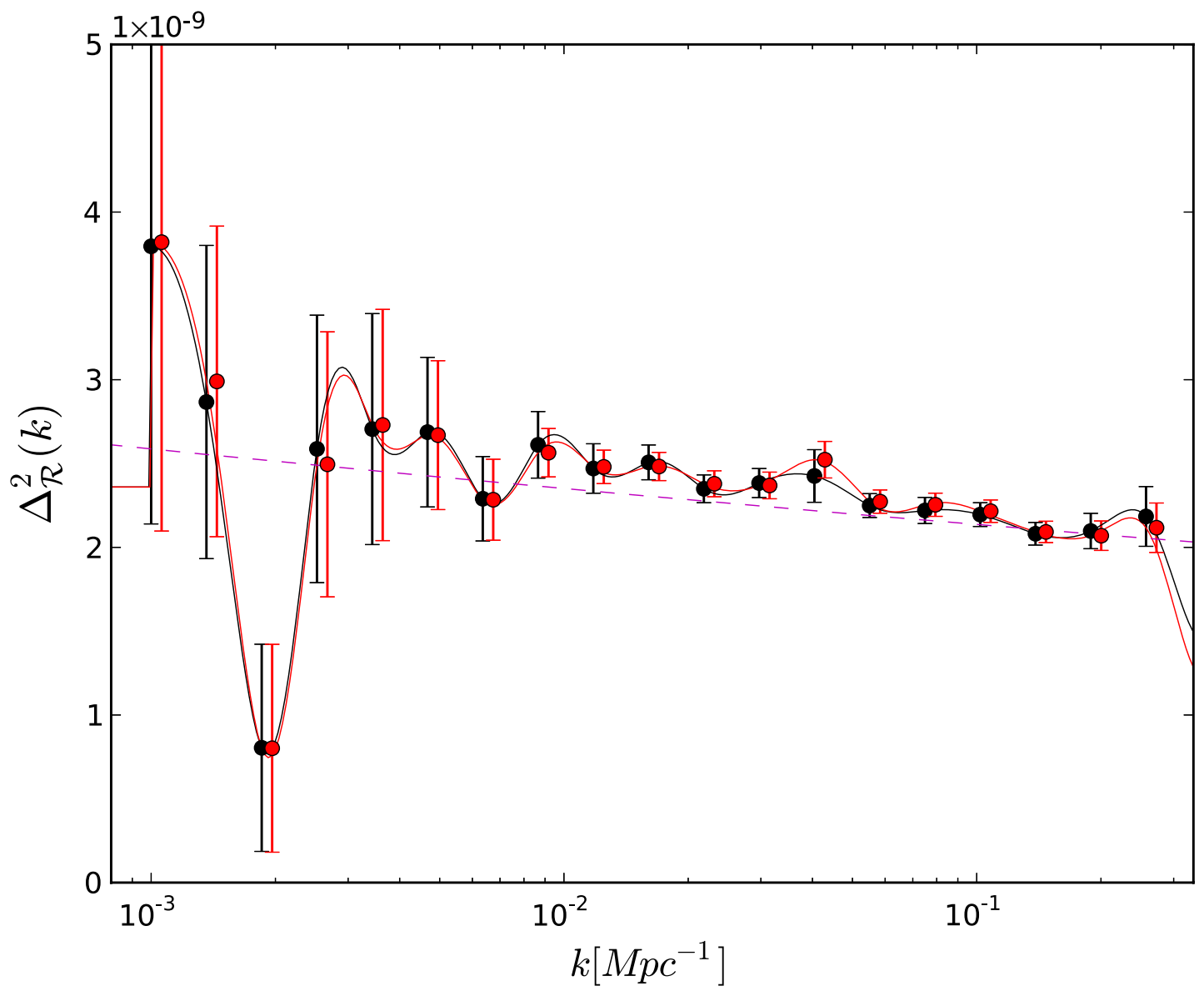

FIG. 2 (color online). The mean PPS node values are shown, including error bars. The black points and error bars indicate the fit to $\mathrm{CMB}$ data only, and the results in light red correspond to CMB + BOSS galaxy clustering (points slightly offset for clarity). The solid lines are the primordial power spectra corresponding to the node values shown. The dashed line is the best-fit power law spectrum to the CMB-only data, marginalized over other parameters.

$p_{i}$ parameters are strongly correlated among themselves, with correlation coefficients up to $|\rho|=0.93$. Moreover, it is not only the nearest neighbors that are strongly correlated (or anticorrelated), but the correlations persist for pairs of nodes well separated in $k$ space.

The reconstructed power spectrum displays no strong evidence for deviations from a power law PPS. The spline PPS model does lead to a better fit to the data, with $\Delta \chi^{2}=33.8$. Given that the spline PPS has 18 parameters more than the power law model, this means that the spline model gives a slightly bigger improvement in the fit to the data than expected purely based on the larger number of free parameters, but the difference is not significant enough to claim strong evidence in favor of the spline model. Some studies have claimed evidence for a dip in the PPS at $k<0.001 \mathrm{Mpc}^{-1}$, but our parametrization does not probe this range because these wave vectors correspond to such large scales that the data are expected to have very little constraining power. We do find a dip in the primordial power spectrum around $k=0.002 \mathrm{Mpc}^{-1}$. This is driven by the deficit in the $\mathrm{CMB}$ temperature power spectrum, relative to the best-fit power-law model, around $\ell \approx 20$.
Figure 3 displays the cosmological parameter constraints both for the free PPS case (for which we discussed the PPS constraints themselves above) using solid black curves/ contours, and for a power law PPS using dashed black curves/contours (we will discuss the results shown in red in the next subsection). The contours show the $68 \%$ and 95\% C.L. regions, while the one-dimensional distributions are the marginalized posterior probability distributions. Allowing more freedom in the PPS relative to a power law spectrum causes both a shift in the best-fit/mean parameter values as well as a widening of the distributions.

For the neutrino mass, the data are consistent with $\Sigma m_{\nu}=0$ for both choices of the PPS, but a free (splined) PPS significantly loosens the upper bound from

$$
\Sigma m_{\nu}<0.63 \mathrm{eV}(\text { power law })
$$

to

$$
\Sigma m_{\nu}<1.9 \mathrm{eV}(\text { spline })
$$

both at $95 \%$ C.L. This is as expected from Fig. 1, where the free PPS could compensate for neutrino mass effects while 


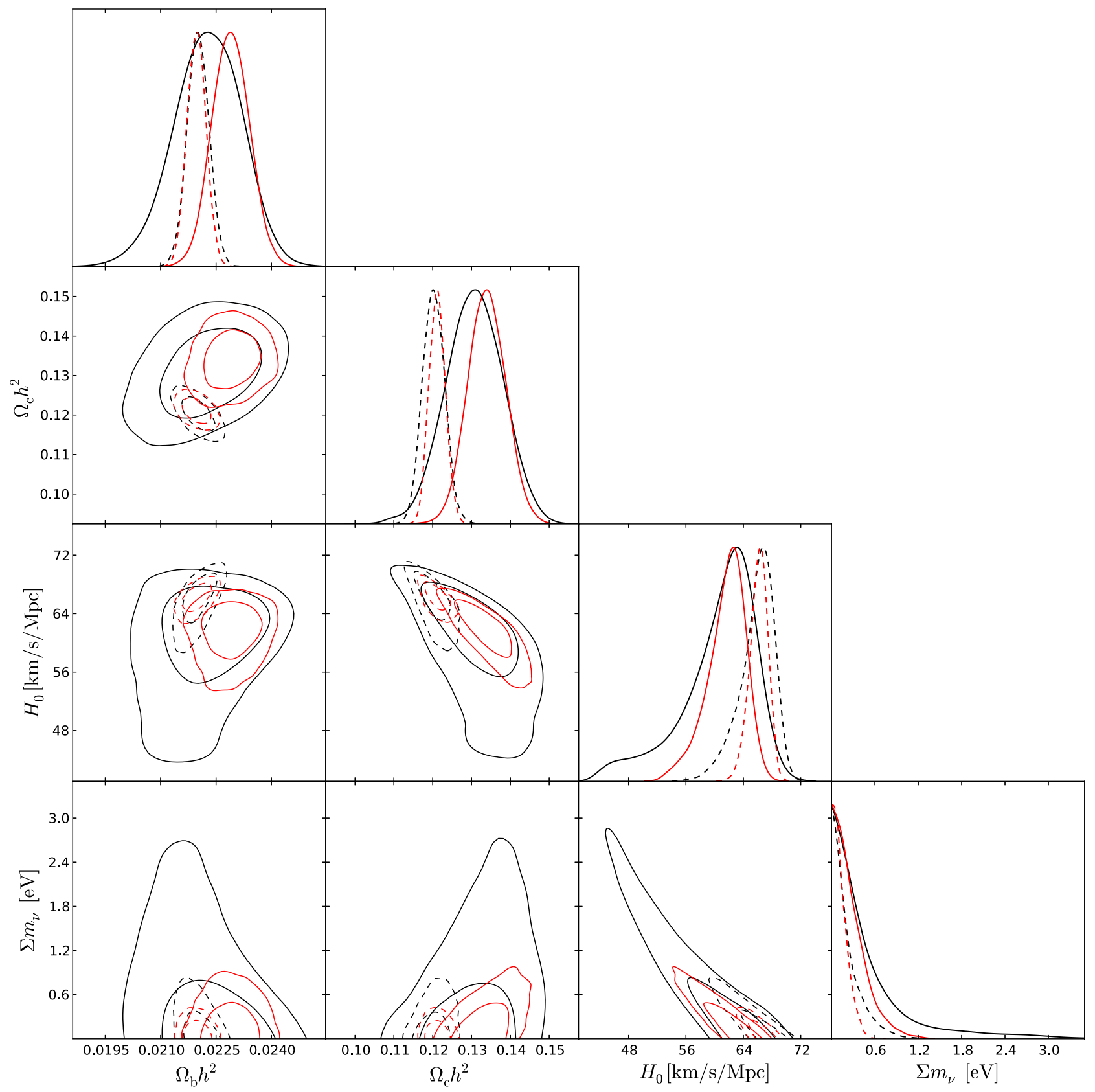

FIG. 3 (color online). The posterior probability distributions of the cosmological parameters, including neutrino mass. Results for the CMB-only data combination are shown in black and those for CMB + BOSS in light red. The solid curves give the results with a free (splined) PPS, while the dashed curves indicate results for the power law case. The number of neutrino species is here fixed to the standard three.

the restricted power law case strongly disfavored larger neutrino masses (despite the ability to adjust noninflationary cosmological parameters).

Let us discuss the constraints in more detail below. In the following, we will often distinguish between "late-universe" parameters on the one hand, $H_{0}$ and $\Sigma m_{\nu}$, and the parameters traditionally constrained very tightly by the acoustic peak structure of the CMB, $\omega_{b}$ and $\omega_{c}$, on the other hand. It is instructive to take the power law case (dashed black curves in Fig. 3) as our starting point. In this case, the PPS is essentially featureless, so that features in the observed CMB spectra directly tell us about the transfer functions, which encode the rich physics of acoustic oscillations during the drag epoch and of the growth of structure at late times, and thus carry a wealth of information on cosmological parameters. Indeed, the CMB peak structure allows for precise measurements of the baryon and cold dark matter densities $\omega_{b}$ and $\omega_{c}$, as shown in Fig. 3. 

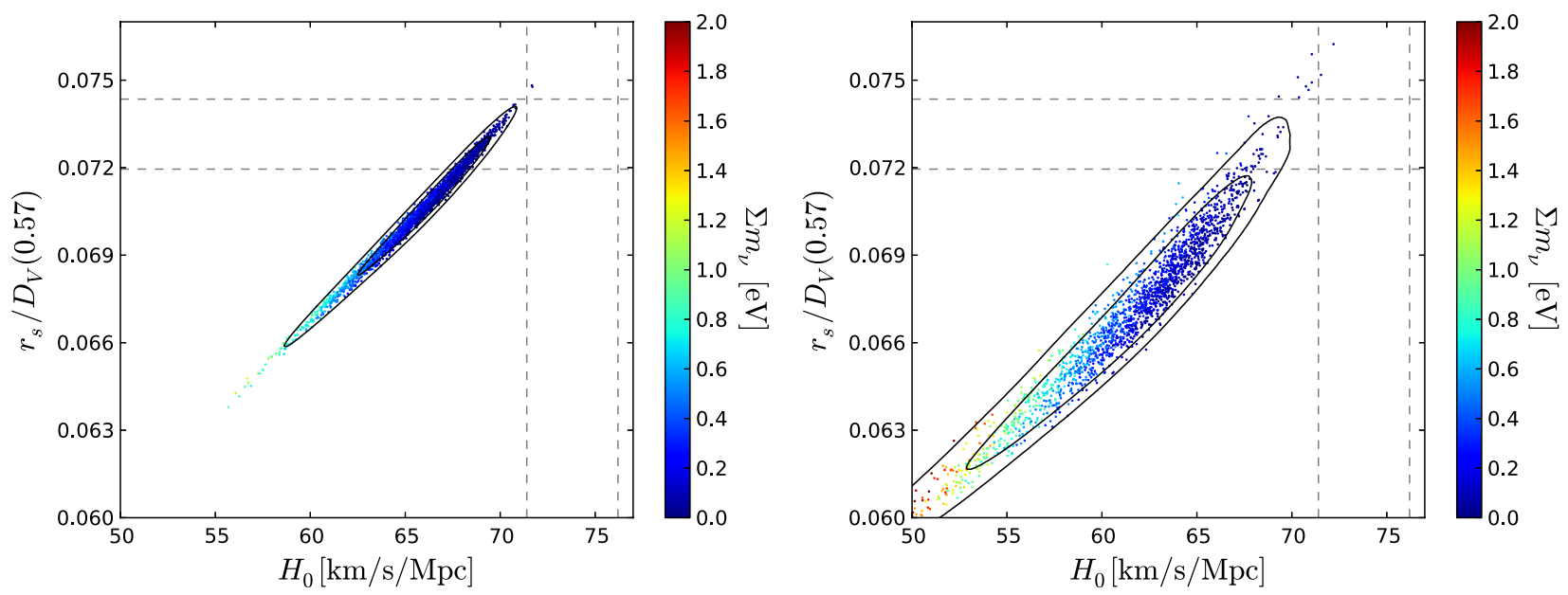

FIG. 4 (color online). Posterior distribution, for the CMB-only data combination, of late-universe "observables" $H_{0}$ and $r_{s} / D_{V}(z=0.57)$, with $\Sigma m_{\nu}$ color coded. Here $r_{s}$ is the sound horizon scale and $D_{V}(z=0.57)$ an effective distance to $z=0.57$, as measured from the angle-averaged BAO scale in the BOSS CMASS sample. The contours indicate $68 \%$ and $95 \%$ confidence regions and the dashed lines indicate $1 \sigma$ ranges from direct measurement of $H_{0}$ and $r_{s} / D_{V}$ (see text). Note that our analysis uses the full shape of the galaxy power spectrum rather than just the BAO measurement. Left: power law PPS. Right: splined PPS. Allowing more freedom in the PPS causes a broadening of the distribution, but retains the strong parameter (anti)correlations so that the addition of a galaxy clustering and/or $H_{0}$ measurement will still tighten the neutrino mass constraint.

Late-universe $(z<1100)$ physics is mainly constrained by the distance to the $\mathrm{CMB}$ last scattering surface and by $\mathrm{CMB}$ lensing, and to a lesser extent by the integrated Sachs-Wolfe (ISW) effect. Still, in the presence of massive neutrinos, significant freedom remains in the parameter direction corresponding to simultaneously varying $\Sigma m_{\nu}$ and $H_{0}$. This degeneracy can be understood in the following simplified picture (see, e.g., [41,60]). Assume that the CMB measures $\omega_{b}$ and $\omega_{c}$ well, independently of neutrino mass. Then, if we increase $\Sigma m_{\nu}$, the main effect on the CMB, ceteris paribus, is a decrease in the distance to the last scattering surface of the $\mathrm{CMB}^{2}$ because neutrino mass increases the neutrino energy density and thus the expansion rate. However, this shift in distance can be compensated by simultaneously lowering the dark energy density $\Omega_{\Lambda}$, and thus $H_{0}$. This explains the degeneracy direction seen in the $H_{0}-\Sigma m_{\nu}$ panel of Fig. 3. When the distance to last scattering is kept constant like this, the joint variation in $\Sigma m_{\nu}$ and $H_{0}$ has a remaining effect on the ISW signal [60] and CMB lensing, so that the $\Sigma m_{\nu}-H_{0}$ degeneracy is not exact and the CMB can still place a meaningful upper limit on neutrino mass. The above explanation is of course only approximate, as in reality there is also some degeneracy with $\omega_{b}$ and $\omega_{c}$.

The late-universe degeneracy of $\Sigma m_{\nu}$ vs $H_{0}$ (or other combinations of late-universe parameters) will play an

\footnotetext{
${ }^{2} \mathrm{~A}$ shift in the distance to CMB last scattering quickly worsens the fit to the data, as the angular size of the sound horizon, which is the ratio of the sound horizon scale and the distance to last scattering, $r_{s} / D_{\text {LSS }}$, is measured very accurately. Note the effect of neutrino mass on $r_{s}$ is small (in the power law PPS scenario, we are in the regime where neutrinos become nonrelativistic after CMB last scattering).
}

important role in understanding how the neutrino and other constraints improve when low-redshift data (galaxy clustering and/or a direct $H_{0}$ measurement) are added. We therefore illustrate this CMB-only degeneracy in Fig. 4. The left panel depicts the power law PPS case. It shows a scatter plot of $H_{0}$ vs the ratio $r_{s} / D_{V}(z=0.57)$, where $r_{s}$ is the sound horizon scale, and $D_{V}(z=0.57)$ the volume-weighted distance to $z=0.57$. For fixed $\omega_{b}$ and $\omega_{c}$, the latter quantity can be derived from $\Sigma m_{\nu}$ and $H_{0}$ (i.e. it is not an independent parameter). The ratio $r_{s} / D_{V}(z=0.57)$ will be useful for analyzing the tightening of constraints when including galaxy clustering in the BOSS CMASS sample, where much of the information comes from the baryon acoustic oscillation (BAO) measurement of $r_{s} / D_{V}(z=0.57)$. The colors indicate the values of $\Sigma m_{\nu}$. The correlation between the three quantities plotted is clear from the figure: increasing $\Sigma m_{\nu}$ leads to decreasing $H_{0}$ and $r_{s} / D_{V}(z=0.57)$. The horizontal and vertical bands indicate the $1 \sigma$ ranges for the measured BAO scale from the BOSS CMASS sample [50] and for $H_{0}$ [56]. We will discuss in the following sections how the BAO measurement relates to the CMASS data used in this work, and how the CMASS data and $H_{0}$ prior help to constrain $\Sigma m_{\nu}$.

Let us now consider what happens when we allow for a splined PPS (right panel of Fig. 4, and solid black line in Fig. 4). The features now allowed in the PPS are partially degenerate with the effect of the transfer functions, thus affecting the cosmological parameter constraints. Specifically, we see that $\omega_{b}$ and $\omega_{c}$ become significantly less tightly constrained and that the mean of (especially) $\omega_{c}$ is shifted to a larger value. Note however that these shifts, and those in the other parameters, are consistent, in the sense that the mean value in the power law scenario is always 
within the range of values allowed by the splined PPS posterior. This makes sense as the power law PPS is effectively embedded within the spline parametrization (one can adjust spline parameters to very closely approximate any power law spectrum). Since the power law case gives a good fit to the data, its mean cosmological parameter values should still give a good fit in the splined PPS scenario.

Moving on to the late-universe parameters, we see that the mean of $H_{0}$ shifts down (in accordance with the anticorrelation with $\omega_{c}$ ) and that $H_{0}$ obtains a larger uncertainty. Finally, the additional PPS freedom allows for larger values of $\Sigma m_{\nu}$. Given the anticorrelation with $H_{0}$ discussed above, this is what would be expected based on both the broadening of the $H_{0}$ posterior and the downward shift of the mean of $H_{0}$. The degeneracy directions of $\Sigma m_{\nu}$ with the other cosmological parameters remain approximately the same when going from a power law to a splined PPS, and the contours are simply widened in all directions (and shifted). For example, as $\Sigma m_{\nu}$ goes up, $H_{0}$ has to decrease to maintain the best possible fit to the CMB data, as in the power law case. Thus, the same physics (e.g. keeping the distance to last scattering constant) still explains the interplay between parameters, but the free PPS allows for larger parameter variations in all directions. This is also shown in the right panel of Fig. 4, which presents the joint constraints on $\Sigma m_{\nu}, H_{0}$, and $r_{s} / D_{V}(z=0.57)$ for the case of a free PPS. This figure will be useful when considering the effect of adding the BOSS and/or $H_{0}$ data.

To summarize the main result of this subsection, the neutrino mass bound from CMB-only data strongly depends on assumptions made about the PPS. If the PPS is restricted to a power law form, a strong upper bound on $\Sigma m_{\nu}$ is obtained. However, taking an agnostic approach with regards to the inflationary specifics of the primordial density fluctuations by allowing for a free form PPS largely undoes the ability of $\mathrm{CMB}$ data to provide detailed information on neutrino mass.

\section{B. CMB + BOSS galaxy power spectrum constraints on neutrino mass}

As mentioned previously, however, the PPS enters differently into the matter density power spectrum, so we now investigate inclusion of the galaxy power spectrum of the BOSS CMASS sample in the data used. The measurements are shown in Fig. 5, with the range used for our analysis colored black. The green dots again indicate the PPS node wave vectors. ${ }^{3}$

\footnotetext{
${ }^{3}$ Note that we show the power spectrum obtained from the data assuming a fiducial cosmology; the inferred spectrum in a different cosmology is a shifted version of the plotted spectrum (both in the horizontal and vertical directions). The mapping between the wave vector in the plot and true wave vector (which is used in the PPS parametrization) is thus cosmology dependent. To allow a direct, but cosmology-dependent, comparison, we have converted the node wave vectors to units $h / \mathrm{Mpc}$, using the value of $h$ in the fiducial cosmology of the BOSS analysis.
}

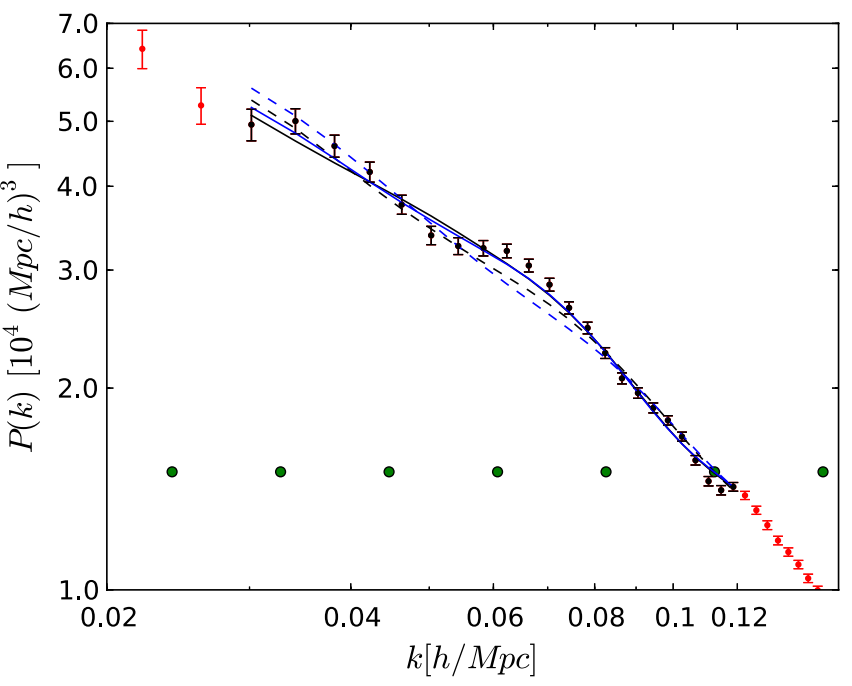

FIG. 5 (color online). The measured galaxy power spectrum of the BOSS CMASS sample is plotted as dots with error bars. The black data points are the only ones used in our analysis, spanning the range $k=0.03-0.12 h \mathrm{Mpc}^{-1}$. The solid curves (black for spline PPS, blue for power law PPS) represent the models that best fit the CMB-only data for fixed $\Sigma m_{\nu}=0$, while the dashed curves give the predictions by the models best fitting the CMB data for $\Sigma m_{\nu}=2.5 \mathrm{eV}$ (same as in Fig. 1). The green dots again indicate the locations of the PPS spline nodes.

For illustration, the solid lines in Fig. 5 show the best-fit spectra to the CMB-only data set for fixed $\Sigma m_{\nu}=0$, i.e. for the same cosmologies as the solid lines in Fig. 1. The dashed lines show the best-fit spectra to the CMB-only data for fixed $\Sigma m_{\nu}=2.5 \mathrm{eV}$ (also as in Fig. 1). While the $\Sigma m_{\nu}=0$ spectra for the two PPS treatments provide a decent fit to the galaxy clustering data and are very similar, increasing neutrino mass to $\Sigma m_{\nu}=2.5 \mathrm{eV}$ significantly worsens the fit in both cases, although less in the case of a free PPS. This suggests that, with the large-scale structure data included, neutrino mass can be constrained meaningfully even in the free (splined) PPS case (although not as well as in the power law PPS scenario); as we have seen in the previous section that is not the case with CMB data only. We will see below that this improvement indeed holds.

The constraints from CMB + BOSS on the spline PPS are shown in red in Fig. 2. For most nodes, both the mean values and uncertainties are very similar to those from CMB only, with only slight improvements in the uncertainties. Thus, the current galaxy clustering data do not have a strong effect on primordial power spectrum constraints.

However, the galaxy power spectrum data do have a strong impact on the cosmological parameter constraints. The posterior distributions from the CMB + BOSS analysis are shown in Fig. 3 in red. In the case of a power law primordial spectrum (dashed), the neutrino mass bound is now 


$$
\Sigma m_{\nu}<0.34 \mathrm{eV} \text { (power law) }
$$

at $95 \%$ C.L., an improvement by almost a factor 2 from the CMB-only case. Note that our bound is slightly stronger than the result $\Sigma m_{\nu}<0.39 \mathrm{eV}$ from combining CMB + BOSS CMASS in [61]; the difference is due to their inclusion of the reconstructed CMB lensing power spectrum from Planck [62], which prefers a larger $\Sigma m_{\nu}$ and thus weakens the upper limit somewhat (see also [41]).

For the free PPS case, we find that the neutrino mass bound from $\mathrm{CMB}+\mathrm{BOSS}$ data becomes

$$
\Sigma m_{\nu}<0.72 \mathrm{eV}(\text { spline })
$$

at $95 \%$ C.L., a factor 2.6 stronger than the CMB-only case. Thus, the galaxy power spectrum is able to rein in the effect of PPS freedom. Indeed this bound is comparable to having no galaxy data but restricting to a power law PPS.

To gain insight into the tightening of the neutrino mass bound, let us first consider the power law PPS case, which is relatively easy to understand. In this case, to a good approximation, the information in the BOSS power spectrum can be represented by a measurement of the $\mathrm{BAO}$ scale, given for the DR9 CMASS sample in [50] by $D_{V}(z=0.57) / r_{s}=$ $13.67 \pm 0.22$. While this measurement ignores any information on the scale-dependent suppression of power due to neutrinos, and is based on a larger range of scales than used in our analysis, we have checked explicitly that the constraints on all parameters from this BAO measurement (combined with $\mathrm{CMB}$ ) are very similar to those using the full galaxy power spectrum (see also, e.g., [61]).

Since $\omega_{b}$ and $\omega_{c}$ are already well measured from CMB only, and are very weakly affected by the BOSS measurement, we focus on the late-universe parameters in Fig. 4 (left panel). The $1 \sigma$ allowed range for $r_{s} / D_{V}(z=0.57$ ) (from now on just $r_{s} / D_{V}$ ) from [50] is shown by the horizontal bands. We have already discussed the CMB-only $\Sigma m_{\nu}-H_{0}$ degeneracy in the previous subsection, and the anticorrelation of $\Sigma m_{\nu}$ with $r_{s} / D_{V}$ can be understood in the same way. An increase in $\Sigma m_{\nu}$ needs to be compensated by a decrease in the dark energy density to keep the distance to CMB last scattering fixed, which in turn leads to a longer distance to $z=0.57$. Since the sound horizon $r_{s}$ is hardly affected when $\Sigma m_{\nu}$ is varied, this leads to a smaller value of the ratio $r_{s} / D_{V}$. It is now obvious from the degeneracy direction shown in the left panel of Fig. 4 and explained above, why the $\mathrm{BAO}$ prior leads to the tightening of the $H_{0}$ posterior, shift upward of its mean, and the strong improvement of the upper bound on $\Sigma m_{\nu}$ seen in Fig. 3. The smaller than expected shift in $H_{0}$ is due to the difference between the $\mathrm{BAO}$ prior and the actual galaxy clustering measurement used in the chains. Indeed, when we replace the galaxy power spectrum measurement with the BAO measurement (not shown), the $H_{0}$ posterior shifts to slightly larger values.

When the PPS is parametrized by a spline (solid curves in Fig. 3), the CMB-only (black) constraints are weaker than in the power law scenario and the inclusion of galaxy clustering data (red) tightens even the $\omega_{b}$ and $\omega_{c}$ posteriors. Regarding the late-universe parameters, the right panel of Fig. 4 shows a similar situation to the power law case (left panel) for the CMB-only data combination: while the allowed ranges of $\Sigma m_{\nu}, H_{0}$, and $r_{s} / D_{V}$ are significantly widened, there is a strong anticorrelation between neutrino mass and $r_{s} / D_{V}$ (and $H_{0}$ ), due to the need for the dark energy density to compensate for the effect of $\Sigma m_{\nu}$ on the distance to last scattering. Treating the galaxy power spectrum measurement as a BAO prior, as we did for the power law PPS, would thus again explain the strong improvement in the neutrino mass bound seen in Fig. 3 and quoted above. In other words, the larger range of allowed $\Sigma m_{\nu}$ values in the splined PPS case, as compared to the power law case, is in large part caused by extending the degeneracy direction with $H_{0}$ and $r_{s} / D_{V}$ (moving further along the diagonal to the bottom left in Fig. 4), and can thus largely be undone by a prior on $r_{s} / D_{V}$ (or $H_{0}$ ).

An important caveat is that, in the splined PPS case, replacing the galaxy power spectrum measurement with a $\mathrm{BAO}$ prior is less justified than in the power law case because, in principle, freedom in the PPS can be (ab)used to mimic or shift the acoustic peak in the galaxy power spectrum, which could result in a completely wrong estimate of $r_{s} / D_{V}$. In practice, the inclusion of CMB data significantly restricts the allowed variations in the PPS so that information on $r_{s} / D_{V}$ is encoded in the galaxy clustering data even with a splined PPS. Indeed, we find that the error bar on $r_{s} / D_{V}$ improves by a factor 2 relative to the CMBonly case when the BOSS data are added (from $r_{s} / D_{v}=$ $0.0662 \pm 0.0037$ to $0.0667 \pm 0.0018$ ). On the other hand, the resulting uncertainty is still about 50\% larger, and the best-fit value significantly smaller, than the direct measurement from [50] $\left(r_{s} / D_{V}=0.0732 \pm 0.0012\right)$.

Based on the above arguments, and since an exact description of the parameter (and PPS) direction(s) constrained by the galaxy power spectrum would be very complex and most likely not that helpful, we simply note that a description in terms of a prior on $r_{s} / D_{V}$ is an insightful approximation and does qualitatively reproduce the effect of the BOSS power spectrum data on the parameter constraints. As in the case of the power law scenario, the BOSS data induce a tightening of the $H_{0}$ and $\Sigma m_{\nu}$ bounds and shift the mean values of $H_{0}$ (slightly) up and the mean value of $\Sigma m_{\nu}$ down, as expected from the degeneracy directions depicted in the right panel of Fig. 4. For comparison, we have also calculated the posteriors that would be obtained if the direct BAO measurement from [50] could be used in the splined PPS case (replacing the galaxy power spectrum measurement) and found that the parameter constraints using the $\mathrm{BAO}$ prior would be significantly stronger than the true constraints from the galaxy power spectrum (e.g. $\Sigma m_{\nu}<0.32 \mathrm{eV}$ instead of $\Sigma m_{\nu}<0.72 \mathrm{eV}$ ), confirming that much of the BAO 
information in the galaxy power spectrum gets lost due to the additional freedom in the PPS and that, unlike in the power law PPS case, treating the galaxy power spectrum measurement as a measurement of the BAO scale is not a quantitatively accurate approximation.

In summary, combining measurements of cosmological perturbations at redshift $z \sim 1100$ and at low redshift $(z \sim 0.57)$ provides valuable information on neutrino mass (and other parameters) even without assuming a form for the primordial power spectrum. The influence of neutrino mass on expansion, rather than the free streaming suppression of the matter power spectrum, is the dominant effect for current large-scale structure data.

\section{C. $\mathrm{CMB}+\mathrm{BOSS}+\mathrm{H}_{\mathbf{0}}$ constraints on neutrino mass}

In the previous sections, and in Figs. 3 and 4, we have explained and shown that $\Sigma m_{\nu}$ is strongly anticorrelated

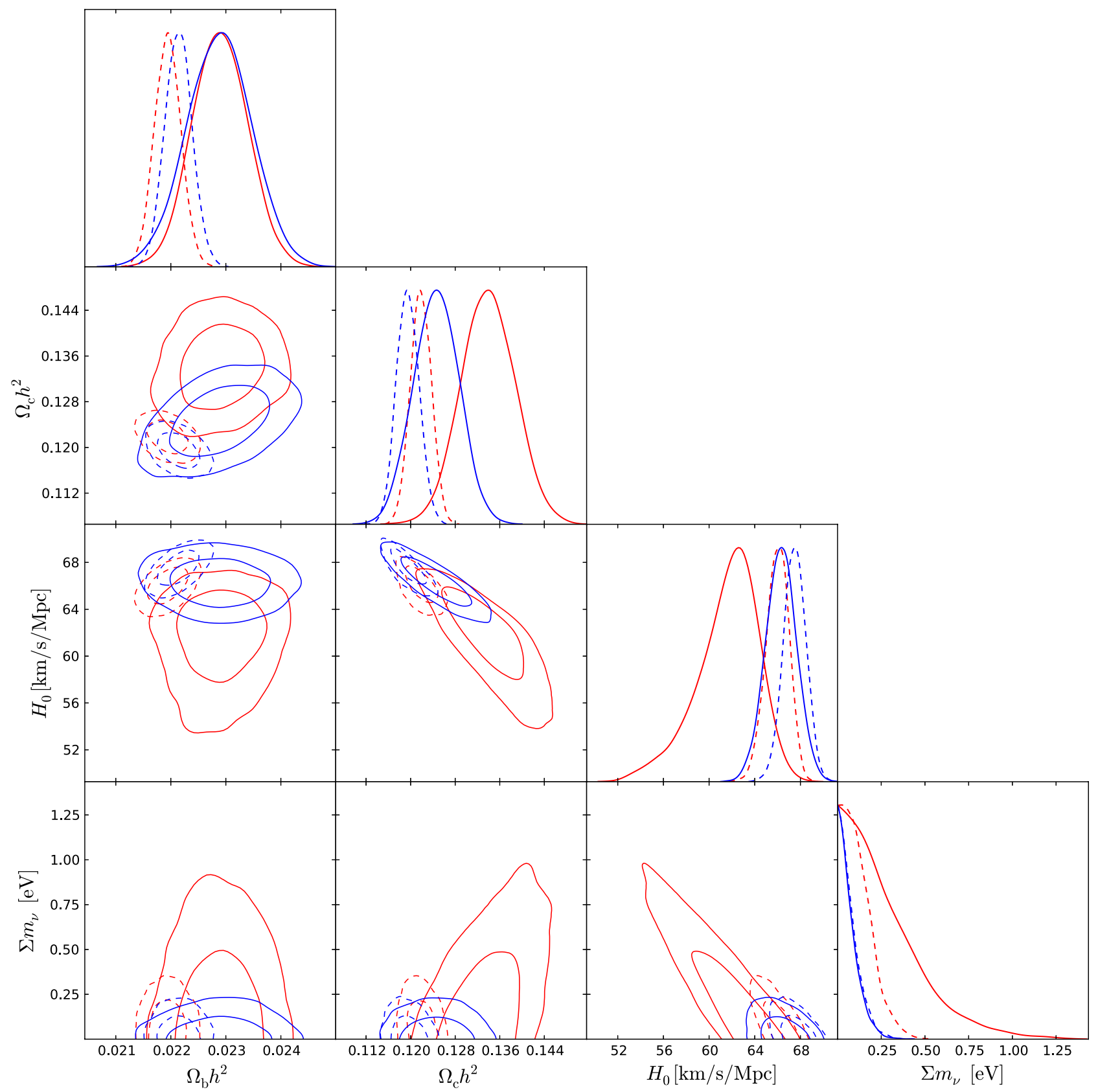

FIG. 6 (color online). The posterior probability distributions of the cosmological parameters, including neutrino mass, when CMB data are combined with low-redshift observations. Note the change of scale from Fig. 3. Results for the CMB + BOSS data combination are shown in light red, while the CMB + BOSS $+H_{0}$ case is shown in dark blue. In both cases, solid curves depict the free (splined) PPS and dashed curves show the power law PPS. The number of neutrino species is here fixed to the standard three. 
with $H_{0}$. Moreover, when including the BOSS data with the CMB data, with a splined PPS the preferred value of the Hubble parameter, $H_{0}=61.6 \pm 2.7 \mathrm{~km} / \mathrm{s} / \mathrm{Mpc}(68 \%$ C.L.), is low compared to the value obtained with the standard power law PPS, $H_{0}=66.1 \pm 1.3 \mathrm{~km} / \mathrm{s} / \mathrm{Mpc}$ (which in turn is slightly lower than the value when $\Sigma m_{\nu}$ is fixed to $0.06 \mathrm{eV}$ ), and even lower compared to the direct HST measurement discussed in Sec. III, $H_{0}=73.8 \pm$ $2.4 \mathrm{~km} / \mathrm{s} / \mathrm{Mpc}$. This means that including the HST $H_{0}$ measurement in our combination of data sets should strongly tighten the upper bound on $\Sigma m_{\nu}$, and especially so for a splined PPS.

Thus, it is worth investigating constraints from the $\mathrm{CMB}+\mathrm{BOSS}+\mathrm{HST}$ data set. We show the results with the blue curves and contours in Fig. 6 and repeat the $\mathrm{CMB}+\mathrm{BOSS}$ results from Fig. 3 in red for comparison. We find that, especially in the splined PPS case, the bounds on $\Omega_{c} h^{2}, H_{0}$, and $\Sigma m_{\nu}$ are all strongly affected. All the changes can be easily understood in terms of the degeneracies between each parameter and $H_{0}$, as shown by the red contours. The neutrino mass upper bound becomes much stronger. In the power law case the upper limit becomes

$$
\Sigma m_{\nu}<0.19 \mathrm{eV}(\text { power law })
$$

while for the spline case it becomes

$$
\Sigma m_{\nu}<0.18 \mathrm{eV}(\text { spline }) .
$$

It is interesting to note that the $H_{0}$ prior is so powerful that the freedom in the PPS in the case of a splined primordial spectrum no longer weakens the neutrino bound.

The prior on $H_{0}$ has little influence on the constraints on the splined PPS itself; those from the CMB + BOSS + HST data combination are similar to those without the HST prior, so we do not show them separately in Fig. 2.

We discussed above that the CMB + BOSS data combination prefers a much lower $H_{0}$ value than the R11 Hubble constant measurement, and that the discrepancy is more severe in the case of a free PPS. This tension between the data sets (in the context of $\Lambda \mathrm{CDM}$ with massive neutrinos) is also reflected in the goodness of fit. When $H_{0}$ is added to the CMB + BOSS data combination, the fit worsens by $\Delta \chi^{2}=10.5$ (using the splined PPS), while $\Delta \chi^{2} \approx 1$ is expected if all data are consistent with a single underlying model. The tight bounds presented above are largely driven by this tension because neutrino mass is anticorrelated with $H_{0}$. Because of this importance of the large value of the $H_{0}$ prior, and because, as discussed briefly in Sec. III, the tension between R11 and Planck might point to the presence of additional errors in the direct $H_{0}$ measurement not included in the published uncertainty, we next study briefly how the $\Sigma m_{\nu}$ bound is affected if the $H_{0}$ measurement is modified.
Replacing the R11 measurement by the revised $H_{0}$ prior of [57] $\left(H_{0}=72.5 \pm 2.5 \mathrm{~km} / \mathrm{s} / \mathrm{Mpc}\right)$, we find the upper limit $\Sigma m_{\nu}<0.21 \mathrm{eV}$, both for a power law and for a splined PPS. The constraint is thus only slightly weakened and it remains true that the inclusion of the Hubble prior renders the neutrino mass limit insensitive to the choice of PPS model. However, if we instead use the value given in [57] based on the maser distance anchor only $\left(H_{0}=70.6 \pm 3.3 \mathrm{~km} / \mathrm{s} / \mathrm{Mpc}\right)$, the neutrino bound weakens to $\Sigma m_{\nu} \lesssim 0.27 \mathrm{eV}$ for both choices of PPS. Since the neutrino mass bound has a non-negligible dependence on which $H_{0}$ value is used, it will be extremely valuable to reach a robust, consensus Hubble constant measurement in the near future.

\section{Summary of neutrino mass bounds}

Table I summarizes the 95\% C.L. upper bounds obtained on $\Sigma m_{\nu}$ for the various combinations of data sets and PPS cases. We see that inflationary freedom strongly affects neutrino mass bounds. Constraining the PPS through multiple types of observations, such as the CMB temperature power spectrum and galaxy power spectrum together, helps considerably. Further adding an external constraint on the Hubble constant compensates almost totally for the added inflationary freedom, allowing a more inflationary model-independent bound.

\section{E. Joint constraints on neutrino mass and number of species}

An important property of neutrinos in addition to their total mass is the effective number of neutrino species (quantifying the energy density of relativistic neutrinos in terms of the energy density of a neutrino that has decoupled completely before electron-positron annihilation). In the standard picture this is $N_{\text {eff }}=3.046$. This can be altered either by adding more species, e.g. sterile neutrinos, or adding more energy density, e.g. by changing the neutrino thermal history or even having other contributions to the (free-streaming) relativistic energy density (in which case the more general term dark radiation applies). Sterile neutrinos in particular have received a lot of recent attention (see [63] and references therein), as the addition of one or two light sterile neutrinos may help explain observed anomalies in short baseline neutrino oscillation experiments. Moreover, $N_{\text {eff }}>3.046$ would ameliorate the tension discussed briefly above between the preferred value of

TABLE I. The $95 \%$ C.L. upper bounds on $\Sigma m_{\nu}$, in $\mathrm{eV}$, are listed for the various combinations of data and theory models. The number of neutrino species is fixed at three.

\begin{tabular}{lccc}
\hline \hline PPS & $\mathrm{CMB}$ & $\mathrm{CMB}+\mathrm{BOSS}$ & $\mathrm{CMB}+\mathrm{BOSS}+H_{0}$ \\
\hline Power law & $\Sigma m_{\nu}<0.63$ & $\Sigma m_{\nu}<0.34$ & $\Sigma m_{\nu}<0.19$ \\
Spline & $\Sigma m_{\nu}<1.9$ & $\Sigma m_{\nu}<0.72$ & $\Sigma m_{\nu}<0.18$ \\
\hline \hline
\end{tabular}




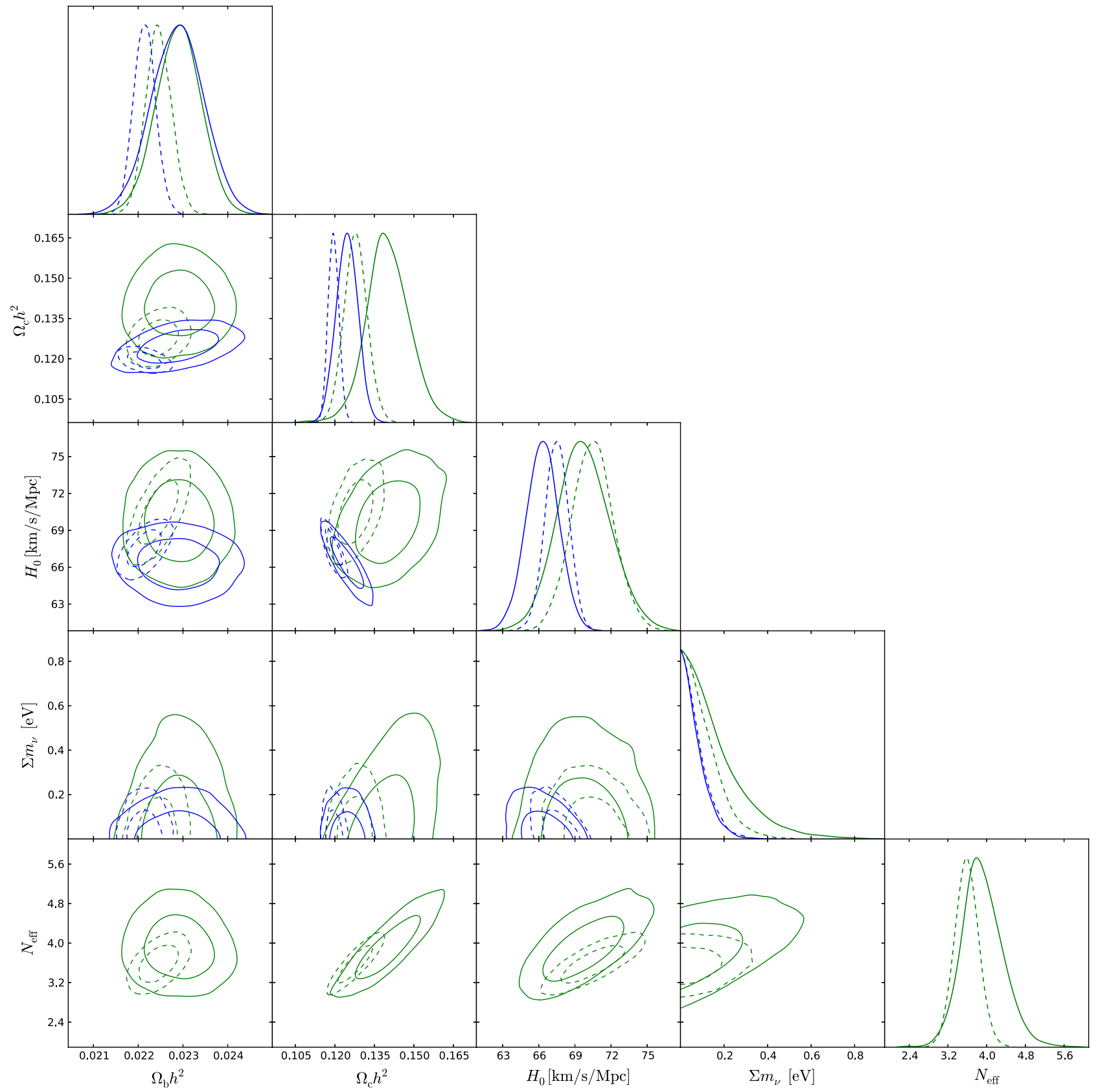

FIG. 7 (color online). The posterior probability distributions of the cosmological parameters for the $\mathrm{CMB}+\mathrm{BOSS}+H_{0}$ data combination. The light green curves and contours present the case with both the total neutrino mass $\Sigma m_{\nu}$ and the effective number of neutrino species $N_{\text {eff }}$ allowed to vary, while the results in dark blue are for the fixed $N_{\text {eff }}=3.046$, also shown in Fig. 6 . Note the change of scale from the previous figures. In both cases, solid curves depict the free (splined) PPS and dashed curves show the power law PPS.

$H_{0}$ from $\mathrm{CMB}$ data and direct measurements of $H_{0}$ (see also [41,64-67]).

We therefore now include $N_{\text {eff }}$ as a free parameter and examine the constraints on its value, ${ }^{4}$ as well as the effect on the neutrino mass bound. When $N_{\text {eff }}$ is a free parameter

\footnotetext{
${ }^{4}$ Big bang nucleosynthesis also constrains $N_{\text {eff }}$, see e.g. [68], but we do not include these data in this study.
}

and the PPS is described by a spline, the freedom in parameter space is so large that we can only obtain robust constraints when all data are combined. We thus only show results for the $\mathrm{CMB}+\mathrm{BOSS}+\mathrm{H}_{0}$ data combination. The green solid (splined PPS) and dashed (power law PPS) curves and contours in Fig. 7 show the posterior distributions with free $N_{\text {eff. }}$. For comparison, we show in blue the results for the same data combination when $N_{\text {eff }}$ is fixed to the standard value. 
TABLE II. The $95 \%$ C.L. upper bounds on $\Sigma m_{\nu}$, in $\mathrm{eV}$, and the mean and standard deviation for $N_{\text {eff }}$, fitting for both simultaneously, are listed for the $\mathrm{CMB}+\mathrm{BOSS}+H_{0}$ combination of data. Recall from Table I the corresponding neutrino mass constraints for fixed $N_{\text {eff }}=3.046$ are $0.19 \mathrm{eV}$ for power law and $0.18 \mathrm{eV}$ for spline PPS.

\begin{tabular}{lc}
\hline \hline PPS & CMB + BOSS $+H_{0}$ for $N_{\text {eff free }}$ \\
\hline Power law & $\Sigma m_{\nu}<0.26, N_{\text {eff }}=3.59 \pm 0.25$ \\
Spline & $\Sigma m_{\nu}<0.43, N_{\text {eff }}=3.92 \pm 0.42$ \\
\hline
\end{tabular}

Table II summarizes the constraints. Looking first at the mean and standard deviation of $N_{\text {eff }}$ (second column), we find that the $\mathrm{CMB}+\mathrm{BOSS}+\mathrm{H}_{0}$ data have a mild preference for $N_{\text {eff }}$ larger than the standard value, at slightly more than $95 \%$ C.L. significance. This is largely driven by the large value of the direct measurement ${ }^{5}$ of $H_{0}$ in combination with the strong correlation between $H_{0}$ and $N_{\text {eff }}$. The splined PPS case prefers a slightly larger $N_{\text {eff }}$ and constrains its value less tightly than the power law PPS case. Also the upper bound on the neutrino mass is weaker for the spline PPS than for a power law, while both are weaker than the bounds obtained for fixed $N_{\text {eff }}=3.046$ (shown in parentheses). Thus, unlike in the case of fixed $N_{\text {eff }}$, when $N_{\text {eff }}$ is a free parameter, even the CMB + BOSS $+H_{0}$ constraints are weakened by allowing additional freedom in the PPS.

The physics behind the $N_{\text {eff }}$ constraint can be understood in the usual way when the PPS follows a power law (e.g. $[41,60])$. In order to fit the CMB temperature power spectrum, an increase in $N_{\text {eff }}$ needs to be accompanied by an increase in $\omega_{c}$ to keep the matter-radiation equality scale constant, and by an increase in $H_{0}$ to keep the angular size of the sound horizon constant (since the increase in $N_{\text {eff }}$ decreases $r_{s}$ ). Moving along this degeneracy direction in parameter space, the dominant remaining effect on the CMB is that the angular size of the Silk damping scale decreases (leading to more damping), making it possible even for CMB-only data to constrain $N_{\text {eff }}$. The strong correlation between $N_{\text {eff }}$ and $H_{0}$ discussed above explains how adding an $H_{0}$ (and galaxy clustering) measurement to the $\mathrm{CMB}$ data strongly tightens the $N_{\text {eff }}$ bound. The above explanation mostly also applies to the splined PPS case, leading to the same approximate parameter degeneracy directions (except for $\omega_{b}$ ), while the extra freedom in the PPS simply broadens (and slightly shifts) the contours. Finally, we note that $N_{\text {eff }}$ and $\Sigma m_{\nu}$ are only weakly correlated with each other.

\footnotetext{
${ }^{5}$ For example, in the power law case, using only CMB data or $\mathrm{CMB}$ with a BAO measurement, the $N_{\text {eff }}$ measurement is consistent with $N_{\text {eff }}=3.046$ at the $95 \%$ C.L. [41].
}

\section{SUMMARY AND CONCLUSIONS}

The universe on large scales provides a unique laboratory for studying fundamental properties of neutrinos. While neutrino mass differences are well measured by more traditional particle physics experiments, the most accurate bound on the absolute neutrino mass scale currently, and for the foreseeable future, comes from cosmological data. It is therefore crucial to investigate to what extent this measurement depends on the assumed cosmological model.

One key ingredient of the assumed cosmology is the primordial power spectrum of curvature perturbations. The strong bounds on neutrino mass quoted in the literature (e.g. $\Sigma m_{\nu}<0.23 \mathrm{eV} \mathrm{[41])} \mathrm{typically} \mathrm{assume} \mathrm{a} \mathrm{power} \mathrm{law}$ PPS (sometimes with a running index). In this article, we have instead studied cosmological neutrino constraints when no functional form is assumed for the PPS. As a compromise between allowing as much freedom in the PPS as possible and computational practicality, we have modeled the PPS by a spline with 20 free nodes (though the results are insensitive to the exact number). We have derived constraints using a compilation of $\mathrm{CMB}$ data and have quantified the effect of including low redshift measurements of the Hubble constant $H_{0}$ and galaxy clustering.

We found that CMB data alone constrains the PPS to better than $10 \%$ over a large range of wave vectors, $k \approx 0.01-0.25 \mathrm{Mpc}^{-1}$, as shown in Fig. 2. No significant deviation from a power law is found. The PPS constraint itself does not improve significantly when current lowredshift data are included.

The constraints on the sum of neutrino masses (Table I) do depend strongly on whether or not low-redshift information is used. For the CMB data set only, $\Sigma m_{\nu}$ is very poorly constrained when the PPS is left free, giving a bound $\Sigma m_{\nu}<1.9 \mathrm{eV}$ (95\% C.L.) compared to $\Sigma m_{\nu}<0.63 \mathrm{eV}$ assuming a power law PPS. However, when low-redshift data are added, the neutrino mass bound becomes stronger and more robust against the choice of the PPS model. Including the galaxy power spectrum from BOSS leads to a bound $\Sigma m_{\nu}<0.72 \mathrm{eV}$ (splined PPS) compared to $\Sigma m_{\nu}<$ $0.34 \mathrm{eV}$ for a power law PPS. Finally, when also a prior on $H_{0}$ from HST is incorporated, the mass limit becomes almost independent of the chosen PPS model, and very strong: $\Sigma m_{\nu} \lesssim 0.18 \mathrm{eV}$.

We also derived joint constraints on neutrino mass and the effective number of neutrino species, $N_{\text {eff }}$, which are summarized in Table II. Combining all three probes, we obtained strong bounds on both quantities, even with a free PPS. Unlike in the case of fixed $N_{\text {eff }}$, the extra freedom in the PPS does weaken the neutrino bounds relative to the power law scenario, by approximately a factor of 1.65 on both the mass and number of species uncertainties. The data show a preference for $N_{\text {eff }}$ larger than the canonical value $N_{\text {eff }}=3.046$, but only at slightly more than 95\% C.L. and strongly driven by the $H_{0}$ measurement. 
In summary, we have found no strong deviations from a power law primordial power spectrum and have shown that, while with a free (splined) PPS, CMB data alone hardly constrain $\Sigma m_{\nu}$, adding galaxy clustering or $H_{0}$ measurements enables strong neutrino limits regardless of the primordial power spectrum model.

\section{ACKNOWLEDGMENTS}

We thank Olga Mena for her assistance with the galaxy power spectrum likelihood code and Jan Hamann for useful discussion regarding Appendix B. Part of the research described in this paper was carried out at the Jet Propulsion Laboratory, California Institute of Technology, under a contract with the National Aeronautics and Space Administration. This work is supported by NASA ATP Grant No. 11-ATP090, DOE Grant No. DE-SC-0007867, and the Director, Office of Science, Office of High Energy Physics, of the U.S. Department of Energy under Contract No. DE-AC0205CH11231, and by Korea World Class University Grant No. R32-2009-000-10130-0. R. d. P. thanks the Institute for the Early Universe at Ewha University, Seoul, where part of this work was performed, for its hospitality.

\section{APPENDIX A: VARYING THE PPS MODEL}

Our goal in this work has been to derive cosmological, and specifically neutrino, constraints when no assumptions are made on the form of the primordial power spectrum. To this end, we have modeled the PPS as a spline with 20 nodes logarithmically spaced in the range $k=0.001-$ $0.35 \mathrm{Mpc}^{-1}$. We chose this number of nodes because it leads to a large amount of freedom in the PPS, allowing for features on approximately the same scales as those induced in the observed power spectra by the transfer functions for matter and radiation perturbations. Moreover, this number of nodes is still small enough for the PPS to be well constrained (to better than $10 \%$ for $k \approx 0.01-0.25 \mathrm{Mpc}^{-1}$ ) and for it to be possible to obtain properly converged $\mathrm{MCMC}$ results.

While our default parametrization is thus well motivated, it is interesting to see how the results change when the number of nodes is varied. We have therefore also calculated constraints using 10 and 40 nodes (with the same $k$ range), using the full $\mathrm{CMB}+\mathrm{BOSS}+H_{0}$ data compilation. The left panel of Fig. 8 shows the mean posterior PPS for these cases (and the best-fit power law spectrum for comparison). As expected, the PPS choices with fewer nodes and hence less freedom approximately follow a smoothed version of the ones with more nodes. While error bars are not explicitly shown to avoid clutter, the uncertainties in the individual node values increase with increasing number of nodes.

The right panel of Fig. 8 shows the resulting posterior distribution of the sum of neutrino masses. As already suggested by the good agreement between the power law and the 20-node spline neutrino limits, the $\Sigma m_{\nu}$ bound is remarkably robust against changes in the assumed PPS model. We do note, however, that the posteriors of other

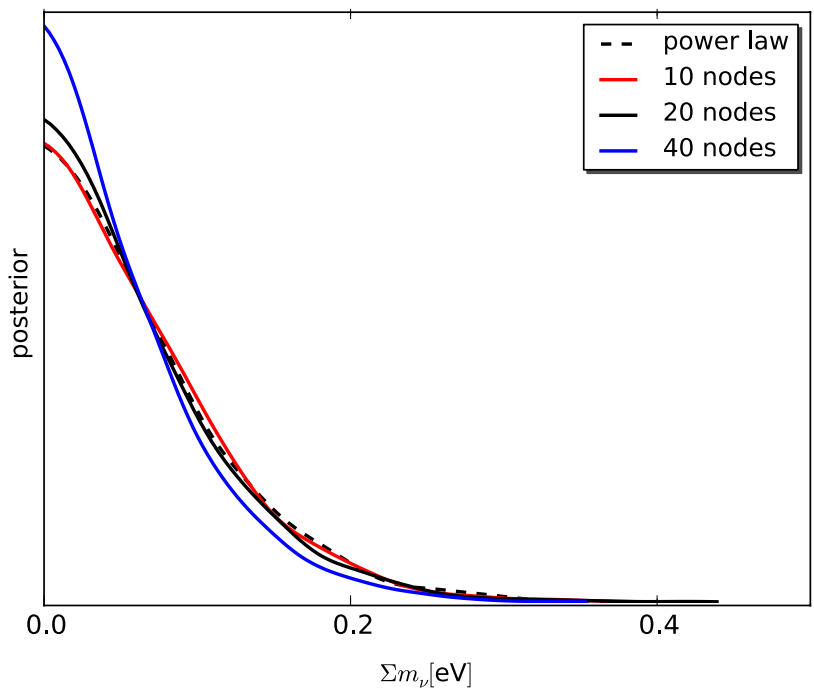

FIG. 8 (color online). Left: mean posterior PPS for different choices of the number of spline nodes (with the best-fit power law PPS shown for comparison), using the full $\mathrm{CMB}+\mathrm{BOSS}+H_{0}$ data combination. The number of neutrino species is fixed to the standard three. Allowing more nodes makes the reconstructed individual PPS node values more noisy (error bars not shown). Right: posterior distribution of the sum of neutrino masses for the cases shown in the left panel. When all data are combined, the bound is remarkably robust against varying assumptions about the PPS and to a first approximation stays constant. The counterintuitive (but small) improvement in the $\Sigma m_{\nu}$ bound as freedom in the PPS is increased can be explained by the fact that the CMB data prefer a lower $H_{0}$ when more freedom in the PPS is allowed, so that adding the higher $H_{0}$ (and galaxy clustering) measurement forces $\Sigma m_{\nu}$ along its degeneracy direction with $H_{0}$, to lower values. 
parameters undergo more significant shifts as the number of nodes is varied. Moreover, the robustness of the neutrino bound relies on the use of low-redshift data to complement the CMB power spectra. The $\Sigma m_{\nu}$ limit depends more strongly on the PPS parametrization when fewer data sets are used.

\section{APPENDIX B: THE ROLE OF MULTIPLE TRANSFER FUNCTIONS AND OF CMB POLARIZATION}

We have seen in this article that access to multiple probes is crucial for obtaining PPS-independent cosmology constraints. When the combined data sets are measurements of cosmic perturbations, here in the form of CMB and galaxy power spectra, this can be understood qualitatively as follows (see, e.g., [38]). An observed power spectrum is the convolution of a transfer function with the primordial power spectrum, with the relevant cosmological (e.g. neutrino) information encoded in the former. If only one power spectrum is observed, the effects of the cosmological parameters are in principle degenerate with variations in the PPS. However, when multiple spectra, with differing transfer functions, are combined, freedom in the PPS can in general not be used to undo the transfer functions effects on all spectra simultaneously and PPS-independent transfer function information can be extracted. As a simple example, if the matter power spectrum could be directly measured at two redshifts, then the ratio of these power spectra would be explicitly independent of the PPS and would give the transfer function of matter perturbations between the two redshifts, leading to constraints on the dark energy density and neutrino mass.
The example of the complementarity described above that we have focused on in this article is the combination of the CMB power spectra with the galaxy power spectrum. In this appendix, we note that even the CMB-only data set makes use of two types of perturbations, namely temperature and E-mode polarization. To see to what extent the inclusion of polarization data has provided PPS-independent cosmological information according to the above description, we have run Monte Carlo chains with the WMAP polarization (WP) data set replaced by a prior on the optical depth to reionization, ${ }^{6} \tau=0.09 \pm 0.013$ (see also [41]). In the power law PPS case, we find that the $\tau$ prior is a good approximation of the information carried by the WP data: the $\Sigma m_{\nu}$ bound only weakens slightly from $\Sigma m_{\nu}<0.63 \mathrm{eV}$ to $\Sigma m_{\nu}<0.83 \mathrm{eV}$. However, for the splined PPS, the neutrino bound weakens by a large amount when the WP data are replaced, going from $\Sigma m_{\nu}<1.9 \mathrm{eV}$ to $\Sigma m_{\nu}<3.2 \mathrm{eV}$. Thus, without the E-mode polarization data, even when $\tau$ is still known as well as it would be with those data, the CMB-only neutrino bound is extremely weak. The polarization data have therefore played a large role in our CMB-only constraints for a free PPS. This is in agreement with our qualitative picture described above of the importance of having access to multiple transfer functions, and bodes well for future data with full polarization information and measurements of galaxy clustering at multiple redshifts.

\footnotetext{
${ }^{6}$ In the case of a free PPS, we have also implemented a prior $\tau=0.097 \pm 0.015$, which is the free-PPS constraint on the optical depth with the WP data included. This choice gives the same neutrino mass bound as the $\tau=0.09 \pm 0.013$ prior.
}

[1] J. Beringer, J.-F. Arguin, R. M. Barnett, K. Copic, O. Dahl, D. E. Groom, C.-J. Lin, J. Lys, H. Murayama, C. G. Wohl et al, Phys. Rev. D 86, 010001 (2012).

[2] W. H. Kinney, Phys. Rev. D 63, 043001 (2001).

[3] D. K. Hazra, A. Shafieloo, and T. Souradeep, Phys. Rev. D 87, 123528 (2013).

[4] G.-B. Zhao, S. Saito, W. J. Percival, A. J. Ross, F. Montesano, M. Viel, D. P. Schneider, M. Manera, J. MiraldaEscudé, N. Palanque-Delabrouille et al., Mon. Not. R. Astron. Soc. 436, 2038 (2013).

[5] M. Archidiacono, E. Giusarma, A. Melchiorri, and O. Mena, Phys. Rev. D 86, 043509 (2012).

[6] R. Easther and R. Flauger, J. Cosmol. Astropart. Phys. 02 (2014) 037.

[7] R. Easther, R. Flauger, P. McFadden, and K. Skenderis, J. Cosmol. Astropart. Phys. 09 (2011) 030.

[8] A. Achucarro, V. Atal, P. Ortiz, and J. Torrado, arXiv:1311.2552.
[9] M. Matsumiya, M. Sasaki, and J. Yokoyama, Phys. Rev. D 65, 083007 (2002).

[10] M. Matsumiya, M. Sasaki, and J. Yokoyama, J. Cosmol. Astropart. Phys. 02 (2003) 003.

[11] N. Kogo, M. Sasaki, and J. Yokoyama, Prog. Theor. Phys. 114, 555 (2005).

[12] R. Nagata and J. Yokoyama, Phys. Rev. D 78, 123002 (2008).

[13] N. Kogo, M. Matsumiya, M. Sasaki, and J. Yokoyama, Astrophys. J. 607, 32 (2004).

[14] G. Nicholson, C. R. Contaldi, and P. Paykari, J. Cosmol. Astropart. Phys. 1 (2010) 016.

[15] A. Shafieloo and T. Souradeep, Phys. Rev. D 70, 043523 (2004).

[16] G. Nicholson and C. R. Contaldi, J. Cosmol. Astropart. Phys. 7 (2009) 011.

[17] G. Goswami and J. Prasad, Phys. Rev. D 88, 023522 (2013). 
[18] P. Hunt and S. Sarkar, J. Cosmol. Astropart. Phys. 01 (2014) 025.

[19] P. Mukherjee and Y. Wang, Astrophys. J. 593, 38 (2003).

[20] P. Mukherjee and Y. Wang, Astrophys. J. 598, 779 (2003).

[21] P. Mukherjee and Y. Wang, Astrophys. J. 599, 1 (2003).

[22] P. Mukherjee and Y. Wang, J. Cosmol. Astropart. Phys. 12 (2005) 007.

[23] S. Leach, Mon. Not. R. Astron. Soc. 372, 646 (2006).

[24] Y. Wang, D. N. Spergel, and M. A. Strauss, Astrophys. J. 510, 20 (1999).

[25] Y. Wang and G. J. Mathews, Astrophys. J. 573, 1 (2002).

[26] S. L. Bridle, A. M. Lewis, J. Weller, and G. Efstathiou, Mon. Not. R. Astron. Soc. 342, L72 (2003).

[27] S. Hannestad, J. Cosmol. Astropart. Phys. 4 (2004) 002.

[28] M. Bridges, A. N. Lasenby, and M. P. Hobson, Mon. Not. R. Astron. Soc. 369, 1123 (2006).

[29] D. N. Spergel, R. Bean, O. Doré, M. R. Nolta, C. L. Bennett, J. Dunkley, G. Hinshaw, N. Jarosik, E. Komatsu, L. Page et al., Astrophys. J. Suppl. Ser. 170, 377 (2007).

[30] M. Bridges, A. N. Lasenby, and M. P. Hobson, Mon. Not. R. Astron. Soc. 381, 68 (2007).

[31] M. Bridges, F. Feroz, M. P. Hobson, and A. N. Lasenby, Mon. Not. R. Astron. Soc. 400, 1075 (2009).

[32] C. Sealfon, L. Verde, and R. Jimenez, Phys. Rev. D 72, 103520 (2005).

[33] L. Verde and H. Peiris, J. Cosmol. Astropart. Phys. 7 (2008) 009.

[34] H. V. Peiris and L. Verde, Phys. Rev. D 81, 021302 (2010).

[35] C. Gauthier and M. Bucher, J. Cosmol. Astropart. Phys. 10 (2012) 050.

[36] S. Hannestad, Phys. Rev. D 63, 043009 (2001).

[37] R. Hlozek, J. Dunkley, G. Addison, J. W. Appel, J. R. Bond, C. Sofia Carvalho, S. Das, M. J. Devlin, R. Dünner, T. Essinger-Hileman et al., Astrophys. J. 749, 90 (2012).

[38] M. Tegmark and M. Zaldarriaga, Phys. Rev. D 66, 103508 (2002).

[39] C. Dvorkin and W. Hu, Phys. Rev. D 82, 043513 (2010).

[40] K. Ichiki and R. Nagata, Phys. Rev. D 80, 083002 (2009).

[41] Planck Collaboration, P. A. R. Ade, N. Aghanim, C. Armitage-Caplan, M. Arnaud, M. Ashdown, F. AtrioBarandela, J. Aumont, C. Baccigalupi, A. J. Banday et al. arXiv:1303.5076; [Astron. Astrophys. (to be published)].

[42] P. A. R. Ade, N. Aghanim, C. Armitage-Caplan, M. Arnaud, M. Ashdown, F. Atrio-Barandela, J. Aumont, C. Baccigalupi, A. J. Banday et al. (Planck Collaboration), arXiv: 1303.5075.

[43] S. Das, T. Louis, M. R. Nolta, G. E. Addison, E. S. Battistelli, J. Bond, E. Calabrese, D. C. M. J. Devlin, S. Dicker, J. Dunkley et al., arXiv:1301.1037.

[44] J. Dunkley, E. Calabrese, J. Sievers, G. E. Addison, N. Battaglia, E. S. Battistelli, J. R. Bond, S. Das, M. J. Devlin, R. Dünner et al., J. Cosmol. Astropart. Phys. 7 (2013) 025.

[45] C. L. Reichardt, L. Shaw, O. Zahn, K. A. Aird, B. A. Benson, L. E. Bleem, J. E. Carlstrom, C. L. Chang, H. M. Cho, T. M. Crawford et al., Astrophys. J. 755, 70 (2012).

[46] R. Keisler, C. L. Reichardt, K. A. Aird, B. A. Benson, L. E. Bleem, J. E. Carlstrom, C. L. Chang, H. M. Cho, T. M. Crawford, A. T. Crites et al., Astrophys. J. 743, 28 (2011).
[47] C. L. Bennett, D. Larson, J. L. Weiland, N. Jarosik, G. Hinshaw, N. Odegard, K. M. Smith, R. S. Hill, B. Gold, M. Halpern et al., Astrophys. J. Suppl. Ser. 208, 20 (2013).

[48] K. S. Dawson, D. J. Schlegel, C. P. Ahn, S. F. Anderson, É. Aubourg, S. Bailey, R. H. Barkhouser, J. E. Bautista, A. Beifiori, A. A. Berlind et al., Astron. J. 145, 10 (2013).

[49] M. White, M. Blanton, A. Bolton, D. Schlegel, J. Tinker, A. Berlind, L. da Costa, E. Kazin, Y.-T. Lin, M. Maia et al., Astrophys. J. 728, 126 (2011).

[50] L. Anderson, E. Aubourg, S. Bailey, D. Bizyaev, M. Blanton, A. S. Bolton, J. Brinkmann, J. R. Brownstein, A. Burden, A. J. Cuesta et al., Mon. Not. R. Astron. Soc. 427, 3435 (2012).

[51] C. P. Ahn, R. Alexandroff, C. Allende Prieto, S. F. Anderson, T. Anderton, B. H. Andrews, É. Aubourg, S. Bailey, E. Balbinot, R. Barnes et al., Astrophys. J. Suppl. Ser. 203, 21 (2012).

[52] A. J. Ross, W. J. Percival, A. G. Sánchez, L. Samushia, S. Ho, E. Kazin, M. Manera, B. Reid, M. White, R. Tojeiro et al., Mon. Not. R. Astron. Soc. 424, 564 (2012).

[53] A. J. Ross, W. J. Percival, A. Carnero, G.-b. Zhao, M. Manera, A. Raccanelli, E. Aubourg, D. Bizyaev, H. Brewington, J. Brinkmann et al., Mon. Not. R. Astron. Soc. 428, 1116 (2013).

[54] M. E. C. Swanson, W. J. Percival, and O. Lahav, Mon. Not. R. Astron. Soc. 409, 1100 (2010).

[55] F.-Y. Cyr-Racine, R. de Putter, A. Raccanelli, and K. Sigurdson, Phys. Rev. D 89, 063517 (2014).

[56] A. G. Riess, L. Macri, S. Casertano, H. Lampeitl, H. C. Ferguson, A. V. Filippenko, S. W. Jha, W. Li, and R. Chornock, Astrophys. J. 730, 119 (2011).

[57] G. Efstathiou, arXiv:1311.3461.

[58] A. Lewis, A. Challinor, and A. Lasenby, Astrophys. J. 538, 473 (2000).

[59] A. Lewis and S. Bridle, Phys. Rev. D 66, 103511 (2002).

[60] Z. Hou, C. L. Reichardt, K. T. Story, B. Follin, R. Keisler, K. A. Aird, B. A. Benson, L. E. Bleem, J. E. Carlstrom, C. L. Chang et al., Astrophys. J. 782, 74 (2014).

[61] E. Giusarma, R. de Putter, S. Ho, and O. Mena, Phys. Rev. D 88, 063515 (2013).

[62] P. A. R. Ade, N. Aghanim, C. Armitage-Caplan, M. Arnaud, M. Ashdown, F. Atrio-Barandela, J. Aumont, C. Baccigalupi, A. J. Banday et al. (Planck Collaboration), arXiv:1303.5077.

[63] K. N. Abazajian, M. A. Acero, S. K. Agarwalla, A. A. Aguilar-Arevalo, C. H. Albright, S. Antusch, C. A. Arguelles, A. B. Balantekin, G. Barenboim, V. Barger et al., arXiv:1204.5379.

[64] M. Wyman, D. H. Rudd, R. A. Vanderveld, and W. Hu, Phys. Rev. Lett. 112, 051302 (2014).

[65] R. A. Battye and A. Moss, Phys. Rev. Lett. 112, 051303 (2014).

[66] J. Hamann and J. Hasenkamp, J. Cosmol. Astropart. Phys. 10 (2013) 044.

[67] S. Gariazzo, C. Giunti, and M. Laveder, J. High Energy Phys. 11 (2013) 211.

[68] K. M. Nollett and G. Steigman, Phys. Rev. D 89, 083508 (2014). 